\title{
LA EXPERIENCIA DE FORMACIÓN CONTINUA PARA LA EDUCACIÓN INFANTIL EN CUBA
}

\section{Olga Franco Garcia*}

Ministério de Educação de Havana-Cuba

\section{Resumen:}

El artículo trata de la experiencia de formación continua para la educación infantil en Cuba. Señala cómo el desarrollo educativo del país es determinante en la preparación profesional de los profesionales de la educación en todos los niveles especialmente en la educación infantil y en función de esta prioridad, realiza una retrospectiva histórica de la formación permanente o continua de los profesionales de la educación infantil en Cuba. Describe como la valoración de la superación continua de los docentes en su evolución se enmarca en un contexto histórico de la Educación Preescolar, estableciendo períodos y etapas, que se distinguen por los hechos históricos y los cambios educacionales ocurridos.

Palabra clave: formación continua; educación infantil; educación en Cuba.

\footnotetext{
*La autora es Master en Ciencias de la Educación, especializada en Educación Preescolar. Su labor la desempeña en el Ministerio de Educación de la República de Cuba, como especialista en el nivel de la Educación Superior Pedagógica. Tiene una experiencia de 47 años en esta propia actividad. Es investigadora y profesora de la maestría del Centro de Referencia de Educación Preescolar para América Latina y el Caribe (CELEP); profesora adjunta de la Universidad de Ciencias Pedagógicas de La Habana; miembro de las secciones de Educación Preescolar y de Juego y Sociedad de la Asociación de Pedagogos de Cuba. Ha participado en todos los congresos internacionales de Pedagogía y Universidad, que se realizan cada dos años en Cuba; es miembro del Comité Organizador del evento Educación Preescolar e Inicial, que se realiza cada año, auspiciado por el CELEP; es autora de programas y planes de estudio para los centros formadores y ha publicado diversos libros de texto para la formación de profesionales de la Educación Preescolar asi como múltiples artículos en diferentes revistas de corte educativo de Cuba y de otros países.

Olh@res, Guarulhos, v. 2, n. 2, p. 08-34. Dezembro, 2014.
} 


\section{Resumo:}

$\mathrm{O}$ artigo trata da experiência em educação continuada para a Educação Infantil em Cuba. Aponta como o desenvolvimento educacional do país é crucial na preparação profissional dos profissionais da educação em todos os níveis, especialmente na educação infantil e de acordo com esta prioridade faz uma retrospectiva histórica da educação continuada dos profissionais educação infantil em Cuba. $\mathrm{O}$ artigo descreve como a avaliação da melhoria contínua de professores em sua evolução está enquadrada num contexto histórico da Educação Infantil, apresentando períodos e etapas, que se distinguem pelos fatos históricos e as mudanças educacionais.

Palavras-chave: formação continuada; Educação Infantil; educação em Cuba. 


\section{Reflexiones generales con una breve y necesaria mirada histórica}

La formación permanente se ha asociado generalmente con la educación de adultos y está orientada en lo fundamental a la preparación del hombre durante toda la vida a partir de las disímiles posibilidades formativas que ofrecen las relaciones sociales que establece con sus colegas, familiares, estudiantes, entre otros.

En la actualidad se jerarquiza el concepto de educación continua o permanente, lo cual demanda una respuesta flexible, variada y consecuente con las exigencias del desarrollo científico técnico que proporcione la adaptación a las condiciones de los cambios que operan en el ámbito laboral. El concepto de educación permanente es inherente al personal docente $\mathrm{y}$, específicamente, la necesidad de una mayor preparación y dominio de los disímiles métodos de enseñanza que se pueden utilizar en los procesos de formación profesional.

La problemática de la formación permanente o continua del docente y su estudio en sentido general ha sido abordada en investigaciones tanto nacionales como internacionales en los últimos años, entre otros autores pueden citarse: M.A. Roschke; M.C. Darini; J. Jadead (1993); R. Forneiro (1996); R. Kent (1996); C. Tunnermann (1996); L. Yarzábal y M. Medina (1996); H. Valdés (1997 y 2002); M. Miguel (1998); V. Arencibia y M. Del Llano (1999); J. Añorga (2001); C. Torres (2007).

Se observan puntos de contacto que, independientemente de los matices, coinciden en ofrecer aportes importantes en cuanto a las vías $\mathrm{y}$ formas que permiten satisfacer las necesidades de perfeccionamiento y preparación de los docentes desde la formación continua en la educación superior, así como el énfasis en la evaluación de la calidad del desempeño, a partir del consenso creciente en relación con el papel determinante de dichos profesionales para impulsar y orientar cambios en los demás agentes del sistema educativo.

Olh@res, Guarulhos, v. 2, n. 2, p. 08-34. Dezembro, 2014. 
Por otra parte la educación continua favorece la integración entre la formación inicial y permanente Autores como, Roschke, M.A; Darini, M.C; Jadead, J han expresado: “...que se remite al trabajo como eje del proceso educativo, fuente de conocimiento y objeto de transformación, que privilegia la participación colectiva $\mathrm{y}$ multidisciplinaria y favorece la construcción dinámica de nuevos conocimientos mediante la investigación, el manejo analítico de la información y el intercambio de saberes y experiencias" ${ }^{2}$

Tunnermann, C precisa lo siguiente: La educación superior tiene que evolucionar de la idea de una educación terminal a la incorporación en su seno del concepto de educación continua o permanente. Esta evolución es una consecuencia de los cambios que se producen en la sociedad contemporánea así como de la reconocida obsolescencia del conocimiento, que se deprecia rápidamente en virtud de su propio dinamismo. Todo esto repercute en el quehacer de las instituciones de la educación superior y las obliga a modificar sus métodos docentes y centrarlos en los procesos de aprendizaje más que en los de enseñanza y en una amplia formación general más que en la demasiado especializada. ${ }^{3}$

Este investigador, en su estudio sobre formación permanente en la educación superior señala: Educación permanente quiere decir que no hay una etapa para estudiar y otra para actuar. Que aprender y actuar forman parte de un proceso existencial que se inicia con el nacimiento y termina con la muerte del individuo. Educación continua quiere decir, no solo poseer conocimientos y las técnicas que permitan desempeñarnos eficientemente en el mundo en que vivimos, sinoundamentalmente, estar capacitados para aprender, reaprender y desaprender permanentemente. ${ }^{4}$

\footnotetext{
${ }^{2}$ Roschke, M.A; Darini, M.C; Jadead, J. (1993). "Educación Permanente y Trabajo en Salud: un proceso en construcción”. En Educ. Med. Y Salud. Vol. 27, No, 4 OPS.

${ }^{3}$ Tunnerman Bernheim, C. (1995). La educación permanente y su impacto en la Educación Superior. UNESCO.

${ }^{4}$ Tunnerman Bernheim, C. (1995). La educación permanente y su impacto en la Educación Superior. UNESCO.
}

Olh@res, Guarulhos, v. 2, n. 2, p. 08-34. Dezembro, 2014. 
La formación continua o permanente del personal docente se organiza en las universidades de ciencias pedagógicas cubanas, conjuntamente con las estructuras de dirección de cada provincia, mediante el perfeccionamiento profesional en cursos de postgrado, entrenamientos y diplomados, así como la formación académica de postgrado mediante especialidades, maestrías y doctorados. Se establecen también otras actividades de superación que responden a necesidades concretas del desarrollo educacional tales como: la autopreparación, la conferencia especializada, el seminario, el taller, el debate científico y otras, entre las que se incluyen las del trabajo metodológico.

Para el desarrollo educacional del país es determinante la preparación profesional de los profesionales de la educación en todos los niveles y en función de esta prioridad la formación permanente o continua está dirigida a:

- La actualización para la renovación y perfeccionamiento de los conocimientos.

- La ampliación para la adquisición de los avances de la ciencia y la técnica y de las habilidades necesarias.

- La especialización para la profundización de los conocimientos y la reorientación por el cambio de especialidad o de funciones profesionales.

Además, la formación continua o permanente asegura:

- La elevación de la efíciencia y calidad del trabajo educacional.

- La adquisición de conocimientos y habilidades de carácter general o específico no recibidos durante la carrera o adquiridos sin profundizar y que son necesarios para la mejor preparación profesional

- La actualización sistemática de los conocimientos y habilidades profesionales de acuerdo con los avances y el desarrollo científicotécnico

Olh@res, Guarulhos, v. 2, n. 2, p. 08-34. Dezembro, 2014. 
- La posibilidad de obtener un nivel más avanzado de profundidad o amplitud de conocimientos y métodos en campos específicos de actuación.

- La profundización o ampliación de conocimientos en áreas particulares, para desarrollar modos de actuación profesional en correspondencia con los avances científico-técnicos.

Se considera, asimismo, que el trabajo de preparación metodológica que se realiza en cada centro educacional se inserta en la amplia concepción de la formación continua o permanente, con un carácter predominantemente grupal, que parte de la definición de las prioridades a atender por cada nivel educacional, desde el propio Ministerio de Educación, en cada curso escolar, que permitan su derivación en cada nivel estructural del sistema y que se materialice en cada grupo en la escuela mediante diferentes actividades, como: las reuniones metodológicas, clases metodológicas, demostrativas o instructivas, clases abiertas, entre otras, en las que predomina el intercambio de experiencias a partir de la preparación previa adquirida.

La formación inicial y continua o permanente se enriquecen con el trabajo metodológico visto como proceso por medio de la actividad y contribuye al desarrollo de modos de actuación profesional pedagógicos en los docentes, y al mismo tiempo debe propiciar la solución de problemas de carácter profesional que afectan la práctica educativa, así como la proyección de estrategias de desarrollo a partir de las condiciones existentes.

Estas posiciones se expresan en cada uno de los niveles educativos con matices particulares. En diferentes países de América Latina y Europa, específicamente: Venezuela, Bolivia, España, Cuba, entre otros se ha estudiado detenidamente las consecuencias que para la educación superior tiene la aceptación de la educación continua y los programas instrumentados son cada vez más una realidad, y responden a las necesidades sociales desde el principio de la igualdad de oportunidades en la educación contemporánea.

Olh@res, Guarulhos, v. 2, n. 2, p. 08-34. Dezembro, 2014. 
En el presente trabajo se comparten los criterios anteriores en relación con la formación o educación continua o permanente a partir de entender la necesidad de profundización y actualización docente en temas particulares que aparecen desde las transformaciones educacionales en las instituciones educativas y específicamente en la universidades, de modo que se reconstruyan sistemáticamente los aprendizajes y estos contribuyan a una mayor calidad en el Sistema Nacional de Educación.

La posición anterior encuentra su génesis en las obras de prestigiosos pedagogos y filósofos cubanos como Félix Varela (1788 1853), José de la Luz y Caballero (1800 - 1862), Manuel Valdés Rodríguez (1849 - 1914), Enrique J. Varona (1849 - 1933), José Martí (1853 - 1895), Alfredo Miguel Aguayo (1866 - 1948), quienes contribuyeron al desarrollo de la teoría y práctica pedagógica y cuyos aportes son aplicables hoy en el contexto educativo de los diferentes subsistemas educacionales sobre la base del reconocimiento de la importancia del estudio y constante actualización del profesor para enseñar y educar.

A partir del año 1959, comienza un nuevo período para el pueblo cubano. Desde los primeros momentos del triunfo revolucionario se promovió una política educativa donde la formación de maestros y profesores y el perfeccionamiento de la preparación de los docentes ya existentes para que pudieran asumir las transformaciones educacionales, pasan a ocupar un lugar importante en el desarrollo educacional.

Desde una nueva perspectiva la preparación del personal docente se desarrolló mediante tres vías fundamentales: la formación emergente y acelerada de maestros y profesores, la continuación de la formación regular que, aunque no daba respuesta masiva constituía lo más estable y estratégico y la utilización del perfeccionamiento y recalificación para elevar el nivel científico-pedagógico de los maestros en ejercicio, titulados y no titulados.

Olh@res, Guarulhos, v. 2, n. 2, p. 08-34. Dezembro, 2014. 
Desde 1990, y hasta la actualidad, la situación se caracteriza por una descentralización de las decisiones en cuanto a las acciones de preparación del personal docente, sobre la base de las necesidades y potencialidades específicas, mediante el vínculo entre los institutos superiores pedagógicos (actualmente denominadas, universidades de ciencias pedagógicas) y las direcciones provinciales de educación, lo cual propició iniciar un trabajo más integrado con el objetivo de elevar la calidad de la educación en los diferentes niveles de enseñanza y la preparación pedagógica desde la formación continua o permanente.

En este período se fortaleció la política de estudios de postgrado, con la creación de un nuevo reglamento, y el proceso de integración entre los Institutos Superiores Pedagógicos y las Direcciones de Educación en sus diferentes niveles, lo cual promovió en mayor cuantía el trabajo metodológico, de superación e investigación en el sector educacional.

El perfeccionamiento sistemático del modelo pedagógico de formación de docentes ha conducido al fortalecimiento de la actividad científico investigativa, pues las transformaciones educacionales exigen de propuestas que se orienten a la validación de los resultados alcanzados.

Las universidades de ciencias pedagógicas, de esta forma, se convierten en centros de altos estudios donde la investigación desempeña un papel fundamental y se dirige hacia la solución de los problemas profesionales pedagógicos que inciden en la formación inicial del profesional de la educación.

De este modo la Educación Superior Pedagógica impone retos a la formación continua del profesorado y directivos, lo cual conduce a la atención, de manera especial, tanto al claustro de profesores de las universidades de ciencias pedagógicas, en su sede central, como a los profesores de las filiales pedagógicas municipales y tutores de los estudiantes.

En sentido general la formación continua o permanente de los docentes de las universidades de ciencias pedagógicas se proyecta hacia el alcance de niveles cualitativamente superiores, desde el punto Olh@res, Guarulhos, v. 2, n. 2, p. 08-34. Dezembro, 2014. 
de vista profesional, lo que permite dar solución a la contradicción actual entre el nivel profesional y científico del claustro de las universidades de ciencias pedagógicas y el reto de formar profesionales sobre la base de asumir la igualdad de oportunidades para todos en las carreras pedagógicas.

La formación permanente o continua de los profesionales de la educación infantil en Cuba

Antes del triunfo de la Revolución, la Educación Preescolar no estaba sistematizada en Cuba. En 1959, no se contaba con personal docente capacitado para dirigir la educación de los niños de cero a seis años, exceptuando a las maestras del grado preescolar que tenían cierta preparación o eran graduadas de las Escuelas Normales de Kindergarten, creadas desde 1902 y cuya exclusividad limitaba tanto el número de ellas como la matrícula, al extremo de que en el año 1952, existían solamente cuatro Escuelas Normales de Kindergarten en el país. No existían estudios superiores de esta especialidad, pues en la Escuela de Pedagogía de la Universidad de La Habana solo se abordaban algunos elementos psicológicos y pedagógicos del niño preescolar.

Con el proceso de creación de condiciones para la apertura de los primeros círculos infantiles comienza a manifestarse la necesidad de capacitar al personal que laboraría en ellos, por lo que en la década de 1960, se asumen variadas alternativas de formación y capacitación del personal técnico y se ponen en práctica los primeros planes de formación regular.

En 1970, surgen las Escuelas de Formación de Educadoras de Círculos Infantiles (EFECI), que ofrecen una preparación especializada tanto teórica como práctica, y que desde el año 1971 quedaron bajo la dirección del recién creado Instituto de la Infancia. En la década de existencia de esta institución se van creando las condiciones para una dirección más científica de la educación de los niños y las niñas de cero a seis años, constituyéndose en un período de Olh@res, Guarulhos, v. 2, n. 2, p. 08-34. Dezembro, 2014. 
despliegue investigativo, que en su conjunto apuntó hacia las distintas esferas que conforman el desarrollo integral. Todo ello, unido al proceso de institucionalización del país ocurre, la integración del Instituto de la Infancia al Ministerio de Educación, en 1980, lo que propició la conformación del subsistema de Educación Preescolar y la total conformación de un Sistema Nacional de Educación. Estos procesos crearon las bases para elevar la preparación del personal docente y la necesidad intrínseca de la formación de nivel superior, ya alcanzada en los restantes subsistemas.

La superación ${ }^{5}$ de los docentes en Cuba ha constituido una política estatal desde el propio triunfo de la Revolución y un momento importante fue en el año 1960 el Ministerio de Educación crea, mediante la Resolución Ministerial 10349 del 20 de abril de 1960, los Institutos Superiores de Educación, más tarde convertidos en Institutos de Superación Educacional (ISE) y después en Institutos de Perfeccionamiento Educacional (IPE) el que entre sus funciones fundamentales tenía asignado realizar cursos, cursillos, seminarios y actividades de índole similar, destinadas a la superación y perfeccionamiento del personal docente, técnico y administrativo en ejercicio.

De esta manera se comenzó a desarrollar por primera vez en Cuba un sistema de actividades de superación con carácter masivo y continuado para todo el personal que prestaba sus servicios en el Ministerio de Educación.

A partir de este año y de forma ininterrumpida, se fue garantizando la superación de los docentes, mediante un sistema de actividades que daba respuesta a las necesidades que demandaba el

\footnotetext{
${ }^{5}$ Nota de la autora. En Cuba denominamos superación de docentes, al proceso continuo y permanente que se desarrolla a lo largo de la vida profesional del docente en ejercicio, y que significa un ilimitado crecimiento personal y humano en el contexto del entorno social en que se desenvuelve, por lo que algunos autores la denominan formación continua o permanente.

El proceso de superación de los docentes, es integral, por lo que forman parte de los aspectos personales, profesionales y sociales. Es entendida la superación, como la educación perenne que permite al docente formar parte de la dinámica del cambio, tanto en su orientación como en el proceso educativo, para enfrentar los problemas planteados por los adelantos científicos y tecnológicos, y los imperativos del desarrollo económico, social y político
}

Olh@res, Guarulhos, v. 2, n. 2, p. 08-34. Dezembro, 2014. 
desarrollo social y la educación, dirigidos tanto a los docentes en ejercicio, como a las estructuras administrativas y de asesoramiento metodológico, hasta la titulación emergente de docentes.

Para realizar el análisis histórico se utilizaron como fuentes principales las tesis de doctorado de Cruz Cruz, C. L. (2007), Escalona Vázquez, I. (2008), Hernández Socarrás, X. (2003), los testimonios de diferentes profesionales de la Educación Preescolar ${ }^{6}$ que durante este período fueron partícipes del proceso de superación continua que acontecía en el territorio y los estudios realizados por Miranda, Avelina (2008) en la determinación de los antecedentes históricos del objeto de investigación, contextualizándose a la particularidad de la superación de los docentes de la Educación Preescolar, (abarca desde 0 a 6 años). Para la realización de este estudio histórico se seleccionaron los siguientes indicadores:

- Concepción de la capacitación y superación de los docentes de la Educación Preescolar. Principales instituciones creadas para la superación.

- Normativas y resoluciones establecidas para la superación de los docentes de la Educación Preescolar.

- Vías de superación de los docentes de la Educación Preescolar.

Los criterios que se han tenido en cuenta para establecer las etapas son: creación de los círculos infantiles, surgimiento de programas de superación y perfeccionamiento de la superación de la Educación Preescolar.

La valoración de la superación continua de los docentes en su evolución se enmarca en un contexto histórico de la Educación Preescolar, por lo que se hace necesario establecer períodos y etapas,

\footnotetext{
${ }^{6}$ Nota de la autora. En Cuba se denomina Educación Preescolar al subsistema que abarca la atención educativa de los niños y niñas desde el nacimiento hasta los 6 años.

Olh@res, Guarulhos, v. 2, n. 2, p. 08-34. Dezembro, 2014.
} 
que se distinguen por los hechos históricos y los cambios educacionales ocurridos.

De esta manera, se asume el período de 1961 hasta la actualidad, atendiendo al criterio de selección y el desarrollo el análisis por etapas:

- I Etapa de 1961- 1970: Concepción inicial de la capacitación y superación de la Educación Preescolar.

- II Etapa de 1971-1991: Institucionalización de la superación en la Educación Preescolar.

- III. Etapa de 1992 hasta la actualidad. - Etapa de perfeccionamiento en la superación de los docentes de la Educación Preescolar.

I Etapa (1961- 1970): Concepción inicial de la superación en los círculos infantiles.

En 1961 por iniciativa del propio Fidel y bajo la dirección de la Federación de Mujeres Cubanas, surgen los primeros círculos infantiles, lo que hace posible que un número considerable de mujeres se incorporaran a la vida laboral y social, mientras que sus hijos eran atendidos en estas instituciones. Estos centros en sus inicios no contaban con personal calificado y generalmente se dedicaban a la atención asistencial de los niños y las niñas.

En relación con los nacientes círculos infantiles, se inicia un proceso organizativo, que parte desde la preparación al personal que atendería la institución, hasta los lineamientos que normarían el trabajo, se confeccionan programas con actividades recreativas y educativas que orientan la labor del personal encargado de atender a los infantes, no obstante, el trabajo de las instituciones continuó siendo un tanto asistencial y conservaba los rasgos de la Escuela Nueva.

Olh@res, Guarulhos, v. 2, n. 2, p. 08-34. Dezembro, 2014. 
Estas contradicciones condujeron a que en el Primer Congreso de Educación y Cultura (1971) se hicieran señalamientos fundamentales de los que no escapó la Educación Preescolar, que indicaba la necesidad de cambios, entre ellos la superación de los docentes que estuvo a cargo de la Dirección Nacional de los Círculos Infantiles (DINCI) y que continuó perfeccionando el Instituto de la Infancia, de reciente creación. Esta institución estableció una acción coordinada con los Ministerios de Educación y de Salud Pública, el entonces Consejo Nacional de Cultura, el Instituto Nacional de Deportes, Educación Física y Recreación (INDER) y otros organismos, con el objetivo de orientar la atención integral del niño y la niña.

En 1962 se encausa la superación de los docentes ya que se crean las Escuelas de Superación Pedagógica y el Instituto de Superación Educacional. Durante esta etapa la superación del personal docente fue una tarea y preocupación constante del Estado, por ello se establecieron cursos para la formación de directoras, asistentes de educación tipo $\mathrm{B}$, dietistas $\mathrm{y}$ de capacitación para enfermeras $\mathrm{y}$ personal de cocina con el objetivo de lograr mayor preparación del personal.

Durante los años 1965 y 1966 surgen las escuelas provinciales de superación para el personal de los círculos infantiles, los cursos eran cortos desde seis meses hasta un año de duración. Como es obvio, las propias características de esta etapa no permiten hacer un estudio detallado de los planes de estudio, pues no se contaba con un perfil o modelo del docente, sólo se poseían los planes y programas, cuya tendencia fundamental era la preparación inicial para enfrentar la actividad laboral.

La superación se ofrecía en las propias instituciones por el personal más preparado y por asesoras regionales.

Como otra vía de superación se funda en 1962 la revista Simientes, órgano divulgativo de la Educación Preescolar que estaba dirigida a los docentes donde se abordaban temas de interés relacionados con los niños de cero hasta los seis años. Olh@res, Guarulhos, v. 2, n. 2, p. 08-34. Dezembro, 2014. 
Se le presta atención a la superación de funcionarios y a los docentes dirigida al trabajo metodológico y una superación a través de cursos de formación emergente, cursos de verano, cursillos, seminarios y al dominio de los programas que imparten de forma general.

En estos primeros años, existía poco personal calificado para la realización del trabajo científico investigativo, originado por la falta de atención a la educación en estas primeras edades. Los especialistas del organismo central realizaban la proyección y orientación de las diferentes actividades de superación; estas aparecían en los documentos principales de organización y planificación del trabajo del MINED y eran ejecutadas por el ISE Nacional y se derivaban hasta las instituciones. Su objetivo general era garantizar la preparación mínima imprescindible de los docentes de la educación.

Se caracterizó, en sentido general por la preparación acelerada del personal que era necesario para los círculos infantiles que ya habían comenzado a fundarse. La superación no era en ese momento el principal propósito para ese personal, pues no se hacía referencia a la continuidad de la preparación, sólo se le prestaba atención a la formación inicial.

Las vías de superación más utilizadas en esta etapa fueron los cursos, seminarios con un período de duración muy corto y dirigido fundamentalmente a objetivos muy generales relacionados con la organización del círculo infantil y la atención al niño.

\section{Etapa (1971-1991): Institucionalización de la superación en la}

\section{Educación Preescolar}

En la etapa comprendida de 1971 hasta 1991 se produjeron importantes cambios en la superación de los docentes de la Educación Preescolar, ya que surgen importantes instituciones dedicadas a la superación de los docentes como la escuela para directoras de los círculos infantiles. En el período que se analiza aumenta el nivel de exigencias a los docentes, por lo que se adquiere una mayor 
preparación técnica y metodológica. El 31 de mayo de 1971 por la Ley 1233 del Consejo de Ministros surge el Instituto de la Infancia, entidad que nace como organismo nacional y marca una nueva etapa en el proceso de superación de los docentes de la Educación Preescolar y el trabajo para el desarrollo de la infancia de 0 a 6 años.

$\mathrm{Su}$ creación significó un reordenamiento estructural que unificó el trabajo de todas aquellas instituciones que se ocupaban de la educación de los niños de estas edades (hogares infantiles y hogares cuna).

Su primera presidenta Vilma Espín definía esta institución expresando que el Instituto de la Infancia era la colaboración estrecha de todos los organismos en la formación del niño, lo más preciado que tiene la sociedad (Ministerio de Educación, Cultura, Salud Pública, y el Instituto Cubano de Radio y Televisión, entre otros, así como distintas organizaciones).

Esta institución realizó un trabajo en todo el proceso de superación de los docentes, así como la planificación, organización y dirección de los diferentes centros infantiles; se concibieron varios programas que fueron puestos en práctica en diferentes momentos: Sistema de Actividades Educativas, a lo que siguió el Proyecto de Programa de Educación y posteriormente se aplicó el Plan de Actividades Educativas.

En 1975 se unificó en un plan único toda la superación para los docentes de la Educación Preescolar y de sus dirigentes, organizados por el Departamento de Capacitación del Instituto de la Infancia, lo que manifiesta la preocupación por la superación del personal para el ejercicio de su práctica. En el gabinete metodológico provincial se ofrecía la titulación del personal en ejercicio y cursos de superación.

Fueron creados los cursos de seis meses para directoras de los círculos infantiles, cursos emergentes de educadoras y ayuda técnica de especialistas soviéticos en el proceso de superación del personal; 
además de la selección de sus mejores graduados para cursar estudios en la antigua URSS, fuerza naciente que a su regreso, con mayor nivel de especialización, se incorpora a los grupos pedagógicos de las Escuelas de Educadoras de Círculos Infantiles, Institutos de Perfeccionamiento Educacional y Círculos Infantiles en los que posteriormente constituyeron grupos de autores en los departamentos de formación del personal docente y el currículo del preescolar para la elaboración de programas y planes de estudio. En estos cursos los docentes se preparaban para dirigir el proceso pedagógico.

Se perfecciona la Revista Simientes como una vía importante de divulgación y superación del personal docente.

La Educación Preescolar bajo la dirección del Instituto de la Infancia logró transformaciones en el orden del crecimiento y la calidad hasta 1980, fecha en que se fusionó con el Ministerio de Educación. Seguidamente se realizaron intentos por hacer más científica la educación, en este sentido va a la profundización del conocimiento de las concepciones teóricas acerca del desarrollo y la educación de los niños y las niñas así como el desarrollo de las investigaciones.

No obstante, esta fue una fructífera etapa en el desarrollo del subsistema de Educación Preescolar y significó un reordenamiento estructural que sirvió para unificar, supervisar y evaluar el trabajo, se acometen determinadas tareas para elevar la calidad de vida de los niños y ampliar la cobertura de atención, se profundiza en sus concepciones y fundamentos mediante las investigaciones, se extienden por el país las aulas de preescolar de las escuelas primarias y los círculos infantiles para los niños y niñas de 5 a 6 años, entre otras.

Se crean los departamentos de Educación Preescolar a nivel de municipio y provincia con el cargo de asesora docente lo cual requirió del perfeccionamiento sistemático del trabajo metodológico el cual queda establecido por primera vez en la RM 220/74 al nivel nacional, provincial, municipal y de centros en el Reglamento que lo establece, 
propiciando que el proceso de superación fuera más planificado, controlado y eficaz.

Precisa además las funciones del metodólogo en su asesoramiento a los departamentos metodológicos municipales y las instituciones que atiende delimitando las responsabilidades y el contenido de la superación dirigida a elevar la calificación profesional de los docentes y dirigentes (muchos en proceso de titulación) para garantizar el objetivo del cumplimiento de las tareas planteadas en el sistema educacional (el estudio de los nuevos programas, indicaciones metodológicas, estudio, generalización y divulgación de experiencias de avanzada, la preparación autodidacta del personal pedagógico y la ayuda individual metodológica que se le presta).

Como parte de los resultados de investigaciones psicológicas y pedagógicas se confeccionó, en 1988, un nuevo Programa de Educación para los círculos infantiles el cual contenía aspectos relacionados con el desarrollo integral y armónico de los niños y las niñas.

Como documentos importantes elaborados que complementan la superación de los docentes aparecen los Planes Nacionales de cursos de postgrado los cuales abordan en sentido general, cuestiones pedagógicas y didácticas necesarias para la dirección del proceso docente educativo.

En esta misma etapa, se establece una nueva concepción de formas de organización de las áreas de desarrollo para los diferentes períodos de edades que componen la etapa de 0-6 años, así como lo relativo a la preparación de las educadoras y maestras en estos contenidos, para lo cual se instrumentaron por el Ministerio de Educación cursos cortos para la formación de especialistas municipales y provinciales por áreas de desarrollo, recibiendo capacitación en contenidos de la Didáctica, Psicología, Pedagogía General, Fisiología, Lenguaje, Fonética y Comunicación, así como, talleres y clases magistrales para demostrar el carácter integral de la enseñanza. 
Esta superación facilitaba su preparación lo cual incidía en los centros que atendían directamente. En esta etapa, sobre todo en su primera mitad, la ayuda prestada por especialistas soviéticos en la superación de personal en centros de estudios de la URSS como medio de colaboración directa por la vía de cursos de postgrado en nuestro país y asesorando al mismo tiempo el trabajo en diferentes esferas, fue de incuestionable valor. Se forman la mayoría de los primeros doctores que ayudan a impulsar la investigación científica.

Como logros de la segunda etapa se expresan los cambios significativos en la Educación Preescolar en la que se elaboran nuevas resoluciones y prioridades para el perfeccionamiento. Se reglamentó el trabajo metodológico dirigido a la superación del personal docente, la actividad científico técnica, investigativa, postgraduada y diplomados.

Como vías fundamentales de superación continúan los cursos emergentes, aunque ya comienzan a introducirse postgrados $\mathrm{y}$ diplomados dirigidos por el Instituto Superior Pedagógico y por especialistas soviéticos, así como la formación de los primeros doctores.

Surgen además importantes instituciones que permitieron una mejor organización y ejecución de la superación de los docentes de las instituciones infantiles. Se desarrollaron actividades importantes donde se evidenció un nivel superior de la superación comparado con la etapa anterior, ya que se elaboraron programas educativos, selección de los mejores graduados para su superación en la antigua URSS, así como la conformación de grupos de autores para la elaboración de programas y planes de estudio.

\section{Etapa (1992-hasta la actualidad): Etapa de perfeccionamiento en la superación de los docentes de la Educación Preescolar.}

La tercera etapa está caracterizada por el desarrollo de la Educación Preescolar cubana y por consiguiente, un mayor perfeccionamiento en el proceso de superación, dado 
fundamentalmente a partir del surgimiento del subsistema de Educación Preescolar e implantación del primer programa científico de educación de esta enseñanza. Si creación del Instituto de la Infancia fue el primer gran salto cualitativo en el desarrollo de la Educación Preescolar revolucionaria, por todas las transformaciones en la elevación de la calidad de este nivel, la instauración del subsistema de educación preescolar como la base del Sistema Nacional de Educación constituye la consolidación de esta educación y del Sistema Nacional de Educación, al erigirse como un sistema único que integra todas las enseñanzas y la formación de especialistas para el desarrollo del país.

Dos hechos significativos suceden en este período, el reforzamiento de la política de postgrado por parte del Ministerio de Educación Superior, con la creación de un nuevo reglamento (Resolución No. 6/1996) y el proceso de integración entre los Institutos Superiores Pedagógicos y las Direcciones de Educación en sus diferentes niveles, lo cual fortaleció la superación. Es a partir de 1992 y 1993 que se realizaron adecuaciones más profundas, que respondieron a los principios del perfeccionamiento de la superación del personal docente. Estas adecuaciones estuvieron dirigidas en primer término, al fortalecimiento de la preparación políticoideológica, por la vía de todas las asignaturas; al reforzamiento de la preparación psicológica y pedagógica para el conocimiento de los niños de las diferentes edades.

La superación abarca a todo el personal docente en ejercicio, su contenido lo determinan las necesidades individuales y los intereses de la institución y el territorio. Sus formas organizativas y modalidades son diversas, según las posibilidades y disponibilidades de los recursos humanos y materiales.

Los docentes que son liberados de su responsabilidad para estudiar no dejan de recibir su salario y esta se realiza sin costo alguno. Los profesores de las universidades de ciencias pedagógicas, del departamento de Educación Preescolar, conjuntamente con los especialistas de este nivel en las direcciones de educación preescolar 
de la provincia, proyectan y ejecutan el sistema de actividades para la superación continua.

Este sistema de superación y perfeccionamiento continuo de los docentes en ejercicio abarca diferentes formas organizativas dentro de ellas la autosuperación, que su contenido responde a las necesidades de cada docente; se realizan cursos dirigidos con la finalidad de completar, profundizar o actualizar los conocimientos y habilidades adquiridas en la formación inicial; estudios de post-grado, diplomados, recalificación desde su puesto de trabajo para el personal que lo requiera, el trabajo metodológico en sus diferentes formas, la investigación y el trabajo científico metodológico; para el personal de dirección, especialistas provinciales, se organizan capacitaciones concentradas a partir de sus necesidades.

Como respuesta al reclamo de los participantes en el Congreso de Pedagogía 95, se crea en 1995 el Centro de Referencia Latinoamericano para la Educación Preescolar (CELEP) con el propósito de promover y coordinar la labor técnica y científica de las ciencias pedagógicas en las edades tempranas y preescolar, de contribuir al perfeccionamiento del nivel profesional de los educadores y de todos aquellos que se relacionan con la educación del niño y la niña en estas edades, así como de las distintas alternativas de educación en una etapa de desarrollo que se considera la más importante y crucial del individuo.

Este centro cumple varios objetivos dentro de los que se destacan el diseño y ejecución de programas de superación posgraduada y de investigación que propicien el enriquecimiento de los conocimientos teóricos y el perfeccionamiento de la práctica pedagógica en la educación inicial y preescolar.

En 1996, se crea la Maestría en Educación Preescolar en el Instituto Pedagógico Latinoamericano y Caribeño (IPLAC). Este hecho amplía las posibilidades de superación del personal docente en ejercicio vinculado a la Educación Preescolar en cualquiera de sus frentes, y enriquece las posibilidades de integración de la formación postgraduada.

Olh@res, Guarulhos, v. 2, n. 2, p. 08-34. Dezembro, 2014. 
En el año 2000 surge la Televisión Educativa donde entre otras actividades se ofertan seminarios nacionales y programas de superación dirigidos por prestigiosos especialistas que aprovechan las ventajas de la televisión y el uso del vídeo. Se elaboran además, tabloides que constituyen materiales significativos para su autosuperación.

Todo lo anterior en función de lograr una mejor preparación de los docentes, que permita enfrentar con mayor éxito las transformaciones que se vienen poniendo en práctica por la dirección de la Revolución, en aras de perfeccionar el sistema educativo cubano.

En el año 2004 se pone en vigor el nuevo Reglamento de la Educación de Postgrado donde establece en su artículo 8 que la superación profesional forma parte de la educación de postgrado y que tiene como objetivo la formación permanente y la actualización sistemática de los graduados universitarios, el perfeccionamiento del desempeño de sus actividades profesionales y académicas, así como el enriquecimiento de su acervo cultural, y en su artículo 7, la educación a distancia como modalidad de superación.

Para una mayor superación de los docentes de la Educación Preescolar, se inicia en el curso 2006-2007 la Maestría en Ciencias de la Educación de amplio acceso, la Mención Preescolar que constituyó un elemento esencial para elevar su superación académica.

Como parte de las transformaciones operadas por el Ministerio de Educación se indica en el curso escolar 2008-2009 el Reglamento del Trabajo Metodológico ( RM 119/08), se señala que constituye un sistema de actividades que de forma permanente, sistémica y sistemática se diseña, ejecuta y controla en los diferentes niveles y tipos de Educación, con el objetivo de garantizar la preparación, pedagógico-metodológica y científica de los docentes egresados y en formación, mediante las direcciones docente-metodológica y científico-metodológica, dirigidas a la conducción eficiente del proceso pedagógico .

Define como direcciones fundamentales del trabajo metodológico, la docente-metodológica y la científico-metodológica. Olh@res, Guarulhos, v. 2, n. 2, p. 08-34. Dezembro, 2014. 
Estas dos direcciones están estrechamente vinculadas entre sí y en la gestión del trabajo metodológico deben integrarse como sistema, en respuesta a los objetivos propuestos. Se establece el asesoramiento y la ayuda metodológica como métodos fundamentales para la superación de los docentes.

Precisa además que el trabajo científico metodológico es la actividad que realizan los educadores con el fin de perfeccionar el proceso pedagógico, mediante proyectos de investigaciones $\mathrm{o}$ utilizando los resultados de investigaciones realizadas, que contribuyan a la formación integral de los educandos y a dar solución a problemas que se presentan en el proceso. Los resultados del trabajo científico metodológico, que se materializa en artículos, ponencias para eventos científicos, libros de texto, monografías, planes y programas de estudio, entre otros, constituyen una de las fuentes principales que le permiten al educador el mejor desarrollo del trabajo docente metodológico .

A modo de conclusión se puede plantear que con el triunfo de la Revolución se producen cambios radicales en la Educación Preescolar, estableciéndose legislaciones ministeriales y orientaciones metodológicas que determinan, según el momento histórico, la formación continua o permanente de los docentes, la que se perfecciona constantemente y se desarrolla con la asesoría de las universidades de ciencias pedagógicas en integración con las direcciones provinciales de Educación.

En este estudio hemos intentado revelar las tendencias fundamentales de la experiencia de formación continua para la educación infantil en Cuba. Revela como la superación de los docentes en sus inicios toma un carácter muy general, predominando la formación del docente para una cultura general pedagógica. Se observan cambios significativos en la Educación Preescolar pues se institucionaliza la formación continua o permanente de los docentes, la cual es diseñada y concebida por las instituciones creadas para ello, lo cual favorece su perfeccionamiento. Se produce un ascenso en su perfeccionamiento a partir del incremento de las diferentes Olh@res, Guarulhos, v. 2, n. 2, p. 08-34. Dezembro, 2014. 
Olga Franco Garcia

modalidades de superación, las cuales se conciben con un carácter más contextualizado y diferenciado.

Olh@res, Guarulhos, v. 2, n. 2, p. 08-34. Dezembro, 2014. 


\section{Bibliografía}

Ambuster Pagán, Romalinda: Reseña histórica de la Educación Preescolar. Editorial Letras Cubanas, Ciudad de La Habana, 1984.

Añorga Morales, J. y otros. (2001). Concepciones teóricas y metodológicas de la Educación. Centro de Postgrado de la U.M.R.P.S.F.X.CH. Sucre.

Añorga Morales, Julia: Glosario de términos de Educación Avanzada, ISP "E. J. Varona”. Ciudad de La Habana, 1995.

Arencibia Sosa, V. et al. (2003). La formación continua a distancia de los profesionales de la educación. Evaluación de su impacto. Curso 17. "Pedagogía 2003”. La Habana.

Arencibia, V. y del Llano M. (1999). La formación inicial y permanente de los profesionales de la educación en los Institutos Superiores Pedagógicos. Curso 53. “Pedagogía 99". La Habana.

Benavides Perera, Zoraida: Trayectoria de la Educación Preescolar en Cuba a partir del Triunfo de la Revolución hasta 1995. En CD Carrera Educación Preescolar 3ra. Versión, 2006. Soporte Electrónico.

Borges Rodríguez, S. (2006). La Educación de las personas con necesidades educativas especiales y su integración social en Cuba. Congreso de Educación y Pedagogía Especial y Primer Simposio Internacional Tecnología, Educación y Desarrollo.

Castellanos Simons, D y otros. (2001). Aprender y enseñar en la escuela. La Habana: Editorial Pueblo y Educación.

Cruz Cruz, Clara Luz: Historia de la Educación Preescolar en la provincia de Villa Clara de 1959 a 1995 - - 1999 Tesis de Maestría - - ISP Félix Varela Villa Clara 1999.

Cruz Cruz, Clara Luz: Sistematización histórica de las transformaciones en el desarrollo de la Educación Preescolar después del Triunfo de la Revolución en la Provincia de Olh@res, Guarulhos, v. 2, n. 2, p. 08-34. Dezembro, 2014. 
Villa Clara. Tesis en Opción al Grado Científico de Doctora en Ciencias Pedagógicas. Villa Clara, 2007.

Forneiro Rodríguez, R. (1996). El sistema de formación inicial y continua del personal docente en Cuba. Taller Internacional Maestro 96. La Habana.

Franco García, Olga. Modelo del profesional del Educador Preescolar Documento rector de la formación del personal pedagógico. MINED 2010

Franco García, Olga. La formación permanente del personal docente en ejercicio. Diferentes modalidades de superación y perfeccionamiento profesional. Papel de los institutos superiores pedagógicos y de las direcciones de educación en cada territorio. En CD Carrera Lic. en educación Preescolar. 2003

Franco García, Olga La formación permanente del personal de la Educación Preescolar. En revista VOCES, No. 1, Septiembre. Uruguay, 1997.

Franco García, Olga En busca de la excelencia profesional del educador preescolar. Un reto a la formación inicial y permanente. Ponencia para el Congreso Pedagogía' 97. La Habana 1997.

Kent, R. (1996). Algunas preguntas sobre la calidad y su evaluación en la Educación Superior Latinoamericana. En Educación Superior con miras al siglo XX. Memorias del Simposio copatrocinado por la UNESCO y el Comité Ejecutivo de la Universidad Bolivariana. (EUB)

MES: Plan nacional de Cursos de Postgrado Año 1979, República de Cuba. MES: Plan Nacional de Estudios de Postgrado. Curso académico 1987-1988. República de Cuba, La Habana, 1987.

MES: Plan Nacional de Superación Profesional de Postgrado Curso Académico 19851986. Ciencias Pedagógicas. República de Cuba.

MIED: ICCP. Índice de los Seminarios Nacionales a dirigentes, metodólogos e inspectores de las Direcciones Provinciales y Direcciones Municipales de Educación 1977-1984. La Habana, 1984.

Olh@res, Guarulhos, v. 2, n. 2, p. 08-34. Dezembro, 2014. 
Miguel Díaz, M. de (1992). "Innovación, Educación y Desarrollo Profesional Docente". En Profesionalización docente y reforma educativa. Austrias: Centro asociado de la UNEED.

Miguel Díaz, M. de (1998). "La evaluación del profesorado universitario. Criterios y propuestas para mejorar la función docente". En Revista de Educación 315. Ciudad Universitaria S.N. Madrid.

MINED: Reglamento de la Educación de Postgrado (R/M 132/04).

MINED: Reglamento del Trabajo Metodológico para el curso 2008- 2009.

Pérez Fleites, Olga Lidia: Modelo de Superación Profesional encaminado a la preparación pedagógica del tutor de los estudiantes de la carrera de Educación Preescolar en condiciones de universalización. Tesis en opción al título de Dra. C. P., UCP "Félix Varela", Villa Clara, 2009.

Roschke, M.A; Darini, M.C; Jadead, J. (1993). "Educación Permanente y Trabajo en Salud: un proceso en construcción”. En Educ. Med. Y Salud. Vol. 27, No, 4 OPS.

Siverio Gómez, Ana María: Reseña histórica de las ideas educativas acerca de la educación preescolar en Cuba. En Reflexiones desde nuestros encuentros. La Habana, 2002.

Torres Batista, César y González Bello. (2007). Formación académica de postgrado en las universidades pedagógicas: retos a la universalización. Curso 24 "Pedagogía 2007”. La Habana.

Tunnerman Bernheim, C. (1995). La educación permanente y su impacto en la Educación Superior. UNESCO.

Tunnerman, Bernheim, C. (1996). La Educación Superior en el Umbral del siglo XXI. Caracas: Ediciones CRESALC, UNESCO. 
Olga Franco Garcia

Yarzábal, L y Medina, M. (1996). "Hacia la transformación de la educación superior en América Latina y el Caribe”. En Educación Superior con miras al siglo XXI. Memorias del Simposio copatrocinado por la UNESCO y el Comité Ejecutivo de la universidad Boliviana (CEUB). Cochabamba.

Olh@res, Guarulhos, v. 2, n. 2, p. 08-34. Dezembro, 2014. 